\title{
RECURRENCE FORMULAS FOR MULTIVARIATE ORTHOGONAL POLYNOMIALS
}

\author{
YUAN XU
}

\begin{abstract}
In this paper, necessary and sufficient conditions are given so that multivariate orthogonal polynomials can be generated by a recurrence formula. As a consequence, orthogonal polynomials of total degree $n$ in $d$ variables that have $\operatorname{dim} \Pi_{n}^{d}$ common zeros can now be constructed recursively. The result is important to the construction of Gaussian cubature formulas.
\end{abstract}

\section{INTRODUCTION}

It is well known that every sequence of univariate orthonormal polynomials $\left\{p_{n}\right\}_{n=0}^{\infty}$ satisfies a three-term relation

$$
x p_{n}(x)=a_{n} p_{n+1}(x)+b_{n} p_{n}(x)+a_{n-1} p_{n-1}(x) .
$$

Moreover, the Favard theorem states that if $\left\{p_{n}\right\}$ satisfies (1.1), then $p_{n}$ is orthonormal if and only if $a_{n}>0$ (cf. [4, 20]). The relation (1.1) is also called the recurrence formula, as it can be rewritten as

$$
p_{n+1}(x)=\frac{1}{a_{n}}\left(x p_{n}(x)-b_{n} p_{n}(x)-a_{n-1} p_{n-1}(x)\right),
$$

and used to define $p_{n}$ recursively. In particular, for given $\left\{a_{n}\right\}$ and $\left\{b_{n}\right\}$ with $a_{n}>0$, one can use (1.2) and $p_{-1}=0$, to generate a sequence of orthonormal polynomials recursively.

For multivariate orthogonal polynomials the situation is much more complicated. To state the corresponding theorem, we need some notations. Let $\mathbb{N}_{0}$ be the set of nonnegative integers. For $n \in \mathbb{N}_{0}$ we denote by $\Pi_{n}^{d}$ the set of polynomials of total degree at most $n$ in $d$ variables, and $\Pi^{d}$ the set of all polynomials in $d$ variables. Let $\mathscr{L}$ be a linear functional defined on $\Pi^{d}$ such that $\mathscr{L}\left(g^{2}\right)>0$ whenever $g \in \Pi^{d}$ and $g \neq 0$. Such an $\mathscr{L}$ is called square positive. For convenience, we assume $\mathscr{L}(1)=1$ throughout this paper. Two polynomials $P$ and $Q$ are said to be orthogonal with respect to $\mathscr{L}$ if $\mathscr{L}(P Q)=0$. For each $n \in \mathbb{N}_{0}$, let $r_{n}^{d}=\operatorname{dim} \Pi_{n}^{d}-\operatorname{dim} \Pi_{n-1}^{d}=\left(\begin{array}{c}n+d-1 \\ n\end{array}\right)$. Throughout this paper, the letter $d$ is reserved for the number of variables or the dimension. It is fixed and will be omitted sometimes.

Received by the editor February 4, 1992 and, in revised form, September 5, 1992 and December 14, 1992.

1991 Mathematics Subject Classification. Primary 42C05, 65D30.

Key words and phrases. Multivariate orthogonal polynomials, recurrence formula, three-term relation, common zeros of multivariate orthogonal polynomials, Gaussian cubature. 
For a sequence of polynomials $\left\{P_{j}^{n}\right\}_{j=1}^{r_{n}}$, where $P_{j}^{n}$ is of total degree $n$, we use the vector notation

$$
\mathbb{P}_{n}(\mathbf{x})=\left[P_{1}^{n}(\mathbf{x}), P_{2}^{n}(\mathbf{x}), \ldots, P_{r_{n}}^{n}(\mathbf{x})\right]^{T} .
$$

For our convenience, we shall write $\left\{\mathbb{P}_{n}\right\}_{n=0}^{\infty}$ instead of $\left\{P_{j}^{n}\right\}_{j=0}^{r_{n}} \infty_{n=0}^{\infty}$. If $P=$ $\left(p_{i j}\right)$ is a matrix whose entries are polynomials in $\Pi^{d}$, we denote by $\mathscr{L}(P)$ the matrix $\left(\mathscr{L}\left(p_{i j}\right)\right)$. We say that $\mathbb{P}_{n}$ is orthogonal if its elements are orthogonal to $\Pi_{n-1}$. The matrix $\mathscr{L}\left(\mathbb{P}_{n} \mathbb{P}_{n}^{T}\right)$ is positive definite if $\mathbb{P}_{n}$ is orthogonal, since $\mathscr{L}$ is square positive, and it is an identity matrix if $\mathbb{P}_{n}$ is orthonormal. Throughout this paper, the $n \times n$ identity matrix is denoted by $I_{n}$, or simply $I$. The notation $A: i \times j$ means that $A$ is a matrix of size $i \times j$. For $\mathbf{x} \in \mathbb{R}^{d}$ we write $\mathbf{x}=\left(x_{1}, \ldots, x_{d}\right)$.

We now state the Favard theorem for the multivariate orthogonal polynomials. This theorem is proved recently in [21,22]; it improves upon a result of Kowalski $[6,7]$ by removing one excessive condition in his theorem.

Theorem 1.1. Let $\left\{\mathbb{P}_{n}\right\}_{n=0}^{\infty}, \mathbb{P}_{0}=1$, be a sequence in $\Pi^{d}$. Then the following statements are equivalent:

(1) There exists a linear functional which is square positive and makes $\left\{\mathbb{P}_{n}\right\}_{n=0}^{\infty}$ an orthonormal basis in $\Pi^{d}$.

(2) For $n \geq 0,1 \leq i \leq d$, there exist matrices $A_{n, i}: r_{n} \times r_{n+1}$ and $B_{n, i}: r_{n} \times$ $r_{n}$ such that the polynomial vectors $\mathbb{P}_{n}$ satisfying the three-term relation

$$
x_{i} \mathbb{P}_{n}=A_{n, i} \mathbb{P}_{n+1}+B_{n, i} \mathbb{P}_{n}+A_{n-1, i}^{T} \mathbb{P}_{n-1}, \quad 1 \leq i \leq d,
$$

where $\mathbb{P}_{-1}=0, A_{-1, i}=0$, and

$$
\operatorname{rank} A_{n}=r_{n+1}, \quad A_{n}=\left(A_{n, 1}^{T}|\cdots| A_{n, d}^{T}\right)^{T} .
$$

This theorem shows that the three-term relation characterizes the orthogonality. However, for $d \geq 2$ this theorem is not as strong as the classical Favard theorem $(d=1)$. Actually, one direction of the theorem says that if there is a sequence of polynomials $\mathbb{P}_{n}$ that satisfies the three-term relation and the rank condition, then it is orthonormal. But it does not answer the question when and which $\mathbb{P}_{n}$ will satisfy such a relation. The rank condition (1.5) implies that there exist matrices $D_{n, i}: r_{n} \times r_{n+1}$ such that

$$
\sum_{i=1}^{d} D_{n, i}^{T} A_{n, i}=I
$$

where we may take $\left(D_{n, 1}^{T}|\cdots| D_{n, d}^{T}\right)$ as the generalized inverse of $A_{n}$. By (1.6) and (1.4) we get

$$
\mathbb{P}_{n+1}(\mathbf{x})=\sum_{i=1}^{d} D_{n, i}^{T} x_{i} \mathbb{P}_{n}(\mathbf{x})-\sum_{i=1}^{d} D_{n, i}^{T} B_{n, i} \mathbb{P}_{n}(\mathbf{x})-\sum_{i=1}^{d} D_{n, i}^{T} A_{n-1, i}^{T} \mathbb{P}_{n-1}(\mathbf{x}),
$$

which is a recurrence formula. However, for given matrices $A_{n, i}$ and $B_{n, i}$, the polynomial sequence $\left\{\mathbb{P}_{n}\right\}$ defined by $(1.7), \mathbb{P}_{-1}=0$, and $\mathbb{P}_{0}=1$, in general does not satisfy the three-term relation, thus, is not orthogonal. Our main result in $\S 2$ gives necessary and sufficient conditions on $A_{n, i}$ and $B_{n, i}$ such that this polynomial sequence is orthogonal. This enables us to define the multivariate 
orthogonal polynomials recursively, which is important in constructing $\left\{\mathbb{P}_{n}\right\}$ that has a maximum number of zeros. Here, zero of $\mathbb{P}_{n}$ means the common zero of the components in $\mathbb{P}_{n}$. It is known that $\mathbb{P}_{n}$ has at most $\operatorname{dim} \Pi_{n-1}$ zeros ([cf. [18]), and the Gaussian cubature of degree $2 n-1$ exists if and only if the corresponding $\mathbb{P}_{n}$ has that many zeros $([13,17])$. We apply our results of $\S 2$ to the construction of Gaussian cubatures in $\S 3$.

\section{ReCURRENCE RELATION}

2.1. Main results. Let $A_{n, i}: r_{n} \times r_{n+1}$ and $B_{n, i}: r_{n} \times r_{n}$ be matrices that satisfy the rank condition (1.5). Let $D_{n, i}^{T}: r_{n+1} \times r_{n}$ be matrices such that $D_{n}^{T}=\left(D_{n, 1}^{T}|\cdots| D_{n, d}^{T}\right)$ is the generalized inverse of $A_{n}$,

$$
D_{n}^{T} A_{n}=I .
$$

We note that (2.1) is equivalent to (1.6). We now define a sequence of polynomials $\left\{\mathbb{P}_{n}\right\}$ by the recurrence formula

$$
\mathbb{P}_{n+1}(\mathbf{x})=\sum_{i=1}^{d} D_{n, i}^{T} x_{i} \mathbb{P}_{n}(\mathbf{x})-E_{n} \mathbb{P}_{n}(\mathbf{x})-F_{n} \mathbb{P}_{n-1}(\mathbf{x}),
$$

where $\mathbb{P}_{-1}=0, \mathbb{P}_{0}=1$, and

$$
E_{n}=\sum_{i=1}^{d} D_{n, i}^{T} B_{n, i} \quad \text { and } \quad F_{n}=\sum_{i=1}^{d} D_{n, i}^{T} A_{n-1, i}^{T} .
$$

Our main result in this section is as follows.

Theorem 2.1. Let $\left\{\mathbb{P}_{k}\right\}_{k=0}^{\infty}$ be defined by (2.2). Then $\left\{\mathbb{P}_{k}\right\}_{k=0}^{\infty}$ satisfies the three-term relation (1.4) if and only if $B_{k, i}$ are symmetric, and

$1^{\circ}$ (rank condition).

$$
\operatorname{rank} A_{k, i}=r_{k}, \quad \operatorname{rank} A_{k}=r_{k+1} ;
$$

$2^{\circ}$ (commuting conditions).

$$
\begin{gathered}
A_{k, i} A_{k+1, j}=A_{k, j} A_{k+1, i}, \\
A_{k, i} B_{k+1, j}+B_{k, i} A_{k, j}=B_{k, j} A_{k, i}+A_{k, j} B_{k+1, i}, \\
A_{k-1, i}^{T} A_{k-1, j}+B_{k, i} B_{k, j}+A_{k, i} A_{k, j}^{T} \\
=A_{k-1, j}^{T} A_{k-1, i}+B_{k, j} B_{k, i}+A_{k, j} A_{k, i}^{T},
\end{gathered}
$$

for $i \neq j, 1 \leq i, j \leq d$, and $k \geq 0$, where $A_{-1, i}=0$.

Moreover, $\left\{\mathbb{P}_{n}\right\}$ forms a basis for $\Pi$.

Combining this result with Theorem 1.1, we can state the following theorem.

Theorem 2.2. Let $\left\{\mathbb{P}_{k}\right\}_{k=0}^{\infty}$ be defined by (2.2). Then there is a linear functional $\mathscr{L}$ that is square positive and makes $\left\{\mathbb{P}_{k}\right\}_{k=0}^{\infty}$ an orthonormal basis for $\Pi^{d}$ if and only if $B_{k, i}$ are symmetric, $A_{k, i}$ satisfy the rank condition (2.4), and together they satisfy the commuting conditions (2.5), (2.6), and (2.7).

This theorem reveals one major difference between univariate and multivariate orthogonal polynomials. Namely, the three-term relation in the multivariate 
case is different from the recurrence relation. For the recurrence formula to generate a sequence of orthogonal polynonmials, its coefficients have to satisfy the commuting conditions. Nevertheless, the following observation seems to be interesting. The commuting conditions are not needed in proving the Favard Theorem, but they are essential for proving that under certain additional conditions on the coefficient matrices, the linear functional in Theorem 1.1 has an integral representation with respect to a nonnegative Borel measure on $\mathbb{R}^{d}[22$, 25]. Actually, the reason that we call (2.5), (2.6), and (2.7) commuting conditions is as follows. For a given sequence of polynomials that satisfies (1.3), we can use the coefficient matrices $A_{n, i}$ and $B_{n, i}$ to define a family of block Jacobi matrices and consider them as linear operators defined on $l^{2}$. These conditions are the ones that make these block matrices commuting. Once these operators commute, we can use the spectral theorem for a commuting family of selfadjoint operators to establish the existence of the measure.

We note that if $\left\{\mathbb{P}_{n}\right\}_{n=0}^{\infty}$ is only orthogonal instead of orthonormal, then $S_{n}^{-1} \mathbb{P}_{n}$ is orthonormal, where $S_{n}$ is a nonsingular matrix that satisfies $S_{n} S_{n}^{T}=$ $\mathscr{L}\left(\mathbb{P}_{n} \mathbb{P}_{n}^{T}\right)$. Therefore, our theorem may be stated in terms of polynomials that are only orthogonal. However, it has to be properly modified since the threeterm relation in this case takes a somewhat different form, with a matrix $C_{n, i}^{T}$, related to $A_{n, i}$ through $S_{n}$, in place of $A_{n-1, i}^{T}$. Also, the commuting conditions become more complicated. Nevertheless, it seems better to look at the orthogonality of multivariate polynomials from the point of view of the polynomial space $\Pi$ being a direct sum of subspaces spanned by polynomials that are components of $\mathbb{P}_{n}$. This is the point of view we have adopted in $[21,22]$.

We shall prove Theorem 2.1 in $\S 2.3$. In the next subsection we give some preliminaries and study properties of orthogonal polynomials. We note that the square positivity of $\mathscr{L}$ is not essential in most results of the next subsection, where it can be replaced by the condition $\mathscr{L}\left(p^{2}\right) \neq 0$.

2.2. Properties of orthogonal polynomials. The basic properties of multivariate orthogonal polynomials are studied in $[21,22,24]$. In particular, if $\left\{\mathbb{P}_{n}\right\}_{n=0}^{\infty}$ is a sequence of orthogonal polynomials, then the coefficient matrices satisfy the rank condition $(2.4)[6,21]$ and the commuting conditions [22]. So the necessity of Theorem 2.1 is known. Here we prove several lemmas that will be used in the proof of the sufficiency. These lemmas are about the properties of the matrices in the three-term relation; they are of some interest in themselves.

For $\alpha=\left(\alpha_{1}, \ldots, \alpha_{d}\right) \in \mathbb{N}_{0}^{d}$ and $\mathbf{x}=\left(x_{1}, \ldots, x_{d}\right) \in \mathbb{R}^{d}$ we write $\mathbf{x}^{\alpha}=$ $x_{1}^{\alpha_{1}} \cdots x_{d}^{\alpha_{d}}$. For $n \in \mathbb{N}_{0}$ we denote by $\mathbf{x}^{n}$ the $r_{n}$-tuple $\left\{\mathbf{x}^{\alpha}\right\}_{|\alpha|=n} \in \mathbb{R}^{r_{n}}$, where the elements are numbered according to the lexicographical order in $\left\{\alpha \in \mathbb{N}_{0}^{d}\right.$, $|\alpha|=n\}$. We let $L_{n, i}$ denote the matrices of size $r_{n-1} \times r_{n}$ that satisfy

$$
L_{n, i} \mathbf{x}^{n}=x_{i} \mathbf{x}^{n-1}, \quad 1 \leq i \leq d .
$$

Clearly, $\operatorname{rank} L_{n, i}=r_{n-1}$, and $\operatorname{rank} L_{n}=r_{n}$, where $L_{n}=\left(L_{n, 1}^{T}|\cdots| L_{n, d}^{T}\right)^{T}$. Moreover, the following identity holds:

$$
L_{n, i} L_{n, i}^{T}=I, \quad 1 \leq i \leq d .
$$

Lemma 2.3. For $n \in \mathbb{N}_{0}, 1 \leq i<j \leq d$, there holds

$$
L_{n, i} L_{n+1, j}=L_{n, j} L_{n+1, i} \text {. }
$$


Proof. From (2.8) it follows that for any $\mathbf{x} \in \mathbb{R}^{d}$

$$
L_{n, i} L_{n+1, j} \mathbf{x}^{n+1}=x_{i} x_{j} \mathbf{x}^{n-1}=L_{n, j} L_{n+1, i} \mathbf{x}^{n+1} .
$$

Since the rank of $L_{n, i} L_{n+1, j}-L_{n, j} L_{n+1, i}$ is at most $r_{n-1}$, the desired identity follows.

We write

$$
\mathbb{P}_{n}=G_{n} \mathbf{x}^{n}+G_{n, n-1} \mathbf{x}^{n-1}+G_{n, n-2} \mathbf{x}^{n-2}+\cdots,
$$

where $G_{n, i}: r_{n} \times r_{i}$ and $G_{n}=G_{n, n}$ is called the leading coefficient of $\mathbb{P}_{n}$. Our next lemma gives the connection between the leading coefficient of orthogonal polynomials and the matrix $A_{n, i}$ in the three-term relation.

Lemma 2.4. If $\left\{\mathbb{P}_{n}\right\}_{n=0}^{\infty}$ satisfies the three-term relation and $A_{n, i}$ satisfy the rank condition (1.5), then for all $n \geq 0, G_{n}$ is invertible. Moreover,

$$
A_{n, i}=G_{n} L_{n+1, i} G_{n+1}^{-1} \text {. }
$$

Proof. If $\mathbb{P}_{n}$ satisfies the three-term relation (1.4), then the coefficient matrices of $\mathbf{x}^{n+1}$ on both sides of the three-term relation are equal. This leads to the identity

$$
A_{n, i} G_{n+1}=G_{n} L_{n+1, i}, \quad 1 \leq i \leq d .
$$

Therefore, it follows that

$$
A_{n} G_{n+1}=\operatorname{diag}\left\{G_{n}, \ldots, G_{n}\right\} L_{n+1} .
$$

We now use induction on $n$. Since $\mathbb{P}_{0}=1$, we have $G_{0}=1$. Suppose that $G_{n}$ has been proved to be invertible. Then $\operatorname{diag}\left\{G_{n}, \ldots, G_{n}\right\}$ is invertible, and from $\operatorname{rank} L_{n+1}=r_{n+1}$ we have

$$
\operatorname{rank}\left(A_{n} G_{n+1}\right)=\operatorname{rank}\left(\operatorname{diag}\left\{G_{n}, \ldots, G_{n}\right\} L_{n+1}\right)=r_{n+1} .
$$

Therefore, by (1.5) and a well-known rank inequality [5, p. 13] we have

$$
\operatorname{rank} G_{n+1} \geq \operatorname{rank}\left(A_{n} G_{n+1}\right) \geq \operatorname{rank} A_{n}+\operatorname{rank} G_{n+1}-r_{n+1}=\operatorname{rank} G_{n+1} .
$$

Thus, it follows that

$$
\operatorname{rank} G_{n+1}=\operatorname{rank}\left(A_{n} G_{n+1}\right)=r_{n+1} .
$$

Hence, $G_{n+1}$ is invertible. The induction is complete. The identity (2.11) follows from (2.12).

Remark. To prove that $G_{n}$ is invertible for a fixed $n$, we need only to assume that $\left\{\mathbb{P}_{k}\right\}$ satisfies the three-term relation for $0 \leq k \leq n-1$.

Our next lemma is a combinatorial identity that gives an interesting relation among the $r_{n}^{\prime} \mathrm{s}$. The lemma is known ([15, p. 8]; I thank Professor H. Schmid for providing me with this reference), but for completeness we give a simple proof here. The idea of this proof will be used in our further development.

Lemma 2.5. For $n \in \mathbb{N}_{0}, d \geq 1$, we have

$$
r_{n}=\sum_{k=1}^{d}(-1)^{k+1}\left(\begin{array}{l}
d \\
k
\end{array}\right) r_{n-k},
$$

where $r_{k}=0$ for $k<0$. 
Proof. Let $\mathscr{N}_{n}=\left\{\alpha \in \mathbb{N}_{0}^{d}:|\alpha|=n\right\}$, and $\mathscr{N}_{n, i}=\left\{\alpha \in \mathbb{N}_{0}^{d}:|\alpha|=n\right.$, and $\left.\alpha_{i} \neq 0\right\}$. Let $\mu(\mathscr{N})$ denote the number of elements in a set $\mathscr{N}$. By counting the number of integer solutions of $|\alpha|=n$, we have $\mu\left(\mathscr{N}_{n}\right)=r_{n}, \mu\left(\mathscr{N}_{n, i}\right)=$ $r_{n-1}, \mu\left(\mathscr{N}_{n, i} \cap \mathscr{N}_{n, j}\right)=r_{n-2}, \mu\left(\mathscr{N}_{n, i} \cap \mathscr{N}_{n, j} \cap \mathscr{N}_{n, k}\right)=r_{n-3}, \ldots$. From $\mathscr{N}_{n}=$ $\mathscr{N}_{n, 1} \cup \cdots \cup \mathscr{N}_{n, d}$ and the inclusion-exclusion principle

$$
\begin{aligned}
\mu\left(\mathscr{N}_{n}\right)= & \sum_{1 \leq i \leq d} \mu\left(\mathscr{N}_{n, i}\right) \\
& -\sum_{1 \leq i \leq j \leq d} \mu\left(\mathscr{N}_{n, i} \cap \mathscr{N}_{n, j}\right)+\cdots+(-1)^{d+1} \mu\left(\mathscr{N}_{n, 1} \cap \cdots \cap \mathscr{N}_{n, d}\right),
\end{aligned}
$$

the identity (2.13) follows.

For any given sequence of matrices $C_{1}, \ldots, C_{d}$, where all $C_{i}$ are of size $s \times t$, we define a matrix $\Xi_{C}: d s \times\left(\begin{array}{l}d \\ 2\end{array}\right) t$ as follows. Let $\Xi_{i, j}: t \times d s, 1 \leq i, j \leq$ $d, i \neq j$, be block matrices defined by

$$
\Xi_{i, j}=\left(\cdots|0| C_{j}^{T}|\cdots|-C_{i}^{T}|0| \cdots\right) ;
$$

i.e., the only two nonzero blocks are $C_{j}^{T}$ at the $i$ th block and $-C_{i}^{T}$ at the $j$ th block. The matrix $\Xi_{C}$ is then defined by using $\Xi_{i, j}$ as blocks in lexicographical order,

$$
\Xi_{C}=\left[\Xi_{1,2}^{T}\left|\Xi_{1,3}^{T}\right| \cdots \mid \Xi_{d-1, d}^{T}\right] .
$$

In particular, we have that $\Xi_{L_{n}}$ is of the size $d r_{n-1} \times\left(\begin{array}{l}d \\ 2\end{array}\right) r_{n}$, and that $\Xi_{L_{n}^{T}}$ is of the size $d r_{n} \times\left(\begin{array}{l}d \\ 2\end{array}\right) r_{n-1}$. We need to know the rank of these two matrices.

Lemma 2.6. For $d \geq 2$ and $n \geq 1$, there holds

$$
\text { rank } \Xi_{L_{n}^{T}}=d r_{n}-r_{n+1} \text {. }
$$

Proof. Since the matrix $\Xi_{L_{n}^{T}}$ is of size $d r_{n} \times\left(\begin{array}{c}d \\ 2\end{array}\right) r_{n-1}$, we shall prove that the dimension of the null space of $\Xi_{L_{n}^{T}}^{T}$ is $r_{n+1}$. Let $Y=\left(Y_{1}^{T}, \ldots, Y_{d}^{T}\right)^{T} \in \mathbb{R}^{d r_{n}}$, where $Y_{i} \in \mathbb{R}^{r_{n}}$. We consider the homogeneous equation in $d r_{n}$ variables

$$
\Xi_{L_{n}^{T}}^{T} Y=0 .
$$

From (2.14) and (2.15) it follows that these equations can be rewritten as

$$
L_{n, i} Y_{j}=L_{n, j} Y_{i}, \quad 1 \leq i<j \leq d .
$$

By (2.8), the elements of $L_{n}, i$ are either 0 or 1. Moreover, there is exactly one 1 in each row, and the rank of $L_{n, i}$ is $r_{n}$. Using the notation $\mathscr{N}_{n}$ and $\mathscr{N}_{n, i}$ in the proof of Lemma 2.5, we can consider $L_{n, i}$ as transforms from $\mathscr{N}_{n}$ to $\mathscr{N}_{n, i}$. We fix a one-to-one correspondence between the elements of $\mathscr{N}_{n}$ and the elements of a vector in $\mathbb{R}^{r_{n}}$, and write $\left.Y_{i}\right|_{\mathcal{F}_{n, j}}=L_{n, j} Y_{i}$. We can then write the linear systems of equations $(2.16)$ as

$$
\left.Y_{i}\right|_{\mathscr{N}_{n, j}}=\left.Y_{j}\right|_{\mathscr{N}_{n, i}}, \quad 1 \leq i<j \leq d .
$$

This gives $\left(\begin{array}{l}d \\ 2\end{array}\right) r_{n-1}$ equations in $d r_{n}$ variables of $Y$, but not all of them are independent. For any distinct integers $i, j$, and $k$, we have

$$
\begin{gathered}
\left.Y_{I}\right|_{\mathscr{N}_{n, j} \cap \mathscr{N}_{n, k}}=\left.Y_{j}\right|_{\mathscr{N}_{n, i} \cap \mathscr{N}_{n, k}},\left.\quad Y_{i}\right|_{\mathscr{N}_{n, k} \cap \mathscr{N}_{n, j}}=\left.Y_{k}\right|_{\mathscr{N}_{n, i} \cap \mathscr{N}_{n, j}}, \\
\left.Y_{j}\right|_{\mathscr{N}_{n, k} \cap \mathcal{N}_{n, i}}=\left.Y_{k}\right|_{\mathscr{N}_{n, j} \cap \mathscr{N}_{n, i}}
\end{gathered}
$$


Therefore, there are exactly $\mu\left(\mathscr{N}_{n, j} \cap \mathcal{N}_{n, k}\right)=r_{n-2}$ duplicated equations among these three systems of equations. Counting all combinations of three different systems of equations in (2.17), we have $\left(\begin{array}{l}d \\ 3\end{array}\right) r_{n-2}$ equations. But among these equations some are counted more than once. Repeating the above argument for four distinct systems of equations in (2.17), we have that there are $\mu\left(\mathscr{N}_{n, i} \cap \mathcal{N}_{n, j} \cap \mathcal{N}_{n, k}\right)=r_{n-3}$ many equations that are duplicated. There are $\left(\begin{array}{l}d \\ 4\end{array}\right)$ combinations of four different systems of equations. We then need to consider five systems of equations, and so on. Therefore, we have that there are

$$
\left(\begin{array}{l}
d \\
3
\end{array}\right) r_{n-2}-\left(\begin{array}{l}
d \\
4
\end{array}\right) r_{n-3}+\cdots+(-1)^{d+1} r_{n-d+1}=\sum_{k=3}^{d}(-1)^{k+1}\left(\begin{array}{l}
d \\
k
\end{array}\right) r_{n-k+1}
$$

duplicated equations in (2.17). Thus, among the $\left(\begin{array}{l}d \\ 2\end{array}\right) r_{n-1}$ equations of (2.16), the number of independent ones is

$$
\left(\begin{array}{l}
d \\
2
\end{array}\right) r_{n-1}-\sum_{k=3}^{d}(-1)^{k+1}\left(\begin{array}{l}
d \\
k
\end{array}\right) r_{n-k+1}=\sum_{k=2}^{d}(-1)^{k}\left(\begin{array}{l}
d \\
k
\end{array}\right) r_{n-k+1} .
$$

Since the dimension of the null space is equal to the number of variables minus the number of independent equations, and there are $d r_{n}$ variables, we have that

$$
\operatorname{dim}\left\{\operatorname{null} \Xi_{L_{n}^{T}}^{T}\right\}=d r_{n}-\sum_{k=2}^{d}(-1)^{k}\left(\begin{array}{l}
d \\
k
\end{array}\right) r_{n-k+1}=\sum_{k=1}^{d}(-1)^{k+1}\left(\begin{array}{l}
d \\
k
\end{array}\right) r_{n+1-k}=r_{n+1},
$$

where the last equality follows from Lemma 2.5 . The proof is complete.

Lemma 2.7. For $d \geq 2$ and $n \geq 1$, there holds

$$
\operatorname{rank} \Xi_{L_{n}}=d r_{n-1}-r_{n-2} \text {. }
$$

Proof. We shall prove that the dimension of the null space of $\Xi_{L_{n}}^{T}$ is $r_{n-2}$. For $Y=\left(Y_{1}^{T}, \ldots, Y_{d}^{T}\right)^{T} \in \mathbb{R}^{d r_{n-1}}$, where $Y_{i} \in \mathbb{R}^{r_{n-1}}$, we consider the homogeneous equation $\Xi_{L_{n}}^{T} Y=0$. By definition, this equation is equivalent to the systems of linear equations

$$
L_{n, i}^{T} Y_{j}=L_{n, j}^{T} Y_{i}, \quad 1 \leq i<j \leq d .
$$

We use $\mathscr{N}_{n}$ and $\mathscr{N}_{n, i}$ as in the proof of the previous lemma. We note that $L_{n, i}^{T} Y_{j}$ is a vector in $\mathbb{R}^{r_{n}}$, whose coordinates corresponding to $\mathscr{N}_{n, i}$ are those of $Y_{j}$ and whose other coordinates are zeros. Thus, the equation (2.18) implies that elements of $Y_{j}$ are nonzero only when they correspond to $\mathscr{N}_{n, j} \cap \mathscr{N}_{n, i}$ in $L_{n, i}^{T} Y_{j}$, that is, $\left.\left(L_{n, i}^{T} Y_{j}\right)\right|_{\mathcal{N}_{n, i} \cap \mathcal{N}_{n, j}}$, and the nonzero elements of $Y_{i}$ are $\left.\left(L_{n, j}^{T} Y_{i}\right)\right|_{\mathscr{N}_{n, i} \cap \mathscr{N}_{n, j}}$. Moreover, these two vectors are equal. Since for any $X \in$ $\mathbb{R}^{r_{n}}$ we have

$$
L_{n-1, j} L_{n, i} X=\left.X\right|_{\mathcal{N}_{n, i} \cap \mathcal{N}_{n, j}},
$$

which follows from $L_{n-1, i} L_{n, j} \mathbf{x}^{n}=x_{i} x_{j} \mathbf{x}^{n-2}$, from (2.9) we have that

$$
\left.\left(L_{n, i}^{T} Y_{j}\right)\right|_{\mathcal{N}_{n, i} \cap \mathcal{N}_{n, j}}=L_{n-1, j} L_{n, i}\left(L_{n, i}^{T} Y_{j}\right)=L_{n-1, j} Y_{j}=\left.Y_{j}\right|_{\mathcal{N}_{n-1, j}} .
$$

Therefore, the nonzero elements of $Y_{i}$ and $Y_{j}$ satisfy

$$
\left.Y_{i}\right|_{\mathscr{N}_{n-1, i}}=\left.Y_{j}\right|_{\mathscr{N}_{n-1, j}}, \quad 1 \leq i<j \leq d .
$$


Thus, there are exactly $\mu\left(\mathscr{N}_{n-1, i}\right)=r_{n-2}$ independent variables in the solution of (2.18). That is, the dimension of the null space of $\Xi_{L_{n}}^{T}$ is $r_{n-2}$.

This lemma will be used in $\S 3$. Our next lemma deals with the singular value decomposition of $A_{n} D_{n}^{T}$.

Lemma 2.8. Let $A_{k, i}$ and $B_{k, i}$ be given matrices such that the $A_{k, i}$ satisfy the rank condition (2.4) and the commuting condition (2.5). Let $\left\{\mathbb{P}_{k}\right\}_{k=0}^{\infty}$ satisfy (2.19) $x_{i} \mathbb{P}_{k}=A_{k, i} \mathbb{P}_{k+1}+B_{k, i} \mathbb{P}_{k}+A_{k-1, i}^{T} \mathbb{P}_{k-1}, \quad 1 \leq k \leq n-1,1 \leq i \leq d$.

Then $Y \in \mathbb{R}^{d r_{n}}$ is in the null space of $I-A_{n} D_{n}^{T}$ if $\Xi_{A_{n-1}^{T}}^{T} Y=0$. Moreover, if $Y=\left(Y_{1}^{T}, \ldots, Y_{d}^{T}\right), Y_{i} \in \mathbb{R}^{r_{n}}$, then $\Xi_{A_{n-1}^{T}}^{T} Y=0$ is equivalent to

$$
A_{n-1, i} Y_{j}=A_{n-1, j} Y_{i}, \quad 1 \leq i<j \leq d .
$$

Proof. The rank condition (2.4) implies that the singular value decomposition of $A_{n}$ is of the form ([5, p. 414])

$$
A_{n}=V_{n}\left(\begin{array}{c}
\Sigma_{n} \\
O
\end{array}\right) W_{n}^{T},
$$

where $\Sigma_{n}: r_{n+1} \times r_{n+1}$ is a nonsingular diagonal matrix, $V_{n}: d r_{n} \times d r_{n}$, and $W_{n}: r_{n+1} \times r_{n+1}$ are orthogonal matrices. Moreover, the columns of $V_{n}$ are the eigenvectors of $A_{n} A_{n}^{T}$. Let $V_{n}=\left(V_{n, 1} \mid V_{n, 2}\right)$, where $V_{n, 1}: d r_{n} \times r_{n+1}$ and $V_{n, 2}: d r_{n} \times\left(d r_{n}-r_{n+1}\right)$. Then by

$$
A_{n} A_{n}^{T}=V_{n}\left[\begin{array}{cc}
\Sigma_{n}^{2} & O \\
O & O
\end{array}\right] V_{n}^{T},
$$

the columns of $V_{n, 2}$ form an orthogonal basis for the null space of $A_{n} A_{n}^{T}$. Since $D_{n}^{T}$ is the generalized inverse of $A_{n}$, we have that $D_{n}^{T}=W_{n}\left(\Sigma_{n}^{-1} \mid O\right) V_{n}^{T}$ and

$$
I-A_{n} D_{n}^{T}=\left(V_{n, 1}, V_{n, 2}\right)\left[\begin{array}{cc}
O & O \\
O & I
\end{array}\right]\left(\begin{array}{c}
V_{n, 1}^{T} \\
V_{n, 2}^{T}
\end{array}\right)=V_{n, 2} V_{n, 2}^{T} .
$$

Therefore, it follows that $Y$ belongs to the null space of $I-A_{n} D_{n}^{T}$ if and only if $V_{n, 2} V_{n, 2}^{T} Y=0$. The last equation is equivalent to $V_{n, 2}^{T} Y=0$, since the columns of $V_{n, 2}$ are orthogonal.

Let $G_{n}$ be the leading coefficient matrix of $\mathbb{P}_{n}$ that satisfies (2.19). From (2.12) and the definition of the matrix $\Xi$ it follows that

$$
\operatorname{diag}\left\{G_{n}^{T}, \ldots, G_{n}^{T}\right\} \Xi_{A_{n-1}^{T}}=\Xi_{L_{n}^{T}} \operatorname{diag}\left\{G_{n-1}, \ldots, G_{n-1}\right\},
$$

where the size of the block diagonal matrix on the left is $d r_{n} \times d r_{n}$, and the one on the right is $\left(\begin{array}{l}d \\ 2\end{array}\right) r_{n-1} \times\left(\begin{array}{l}d \\ 2\end{array}\right) r_{n-1}$. Therefore, by Lemma 2.4 , the remark that follows it, and Lemma 2.6 we have

$$
\text { rank } \Xi_{A_{n-1}^{T}}=d r_{n}-r_{n+1} \text {. }
$$

From (2.5), (2.14), and (2.15), it follows that $A_{n}^{T} \Xi_{A_{n-1}^{T}}=0$, which, by (2.1), is equivalent to

$$
A_{n} A_{n}^{T} \Xi_{A_{n-1}^{T}}=0 .
$$

Therefore, the columns of $\Xi_{A_{n-1}^{T}}$ belong to the null space of $A_{n} A_{n}^{T}$. Since $\operatorname{rank} A_{n} A_{n}^{T}=\operatorname{rank} A_{n}=r_{n+1}$, the rank condition (2.20) shows that the columns 
of $\Xi_{A_{n-1}^{T}}$ actually span this null space. Thus, there exists a matrix $Q_{n}:\left(\begin{array}{l}d \\ 2\end{array}\right) r_{n-1} \times$ $\left(d r_{n}-r_{n+1}\right)$, whose columns are linearly independent, such that $V_{n, 2}=\Xi_{A_{n-1}^{T}} Q_{n}$. Therefore, $V_{n, 2}^{T} Y=0$ if $\Xi_{A_{n-1}^{T}}^{T} Y=0$.

Corollary 2.9. Let $d=2$ and $n \geq 1$. Then $Y=\left(Y_{1}^{T}, Y_{2}^{T}\right), Y_{i} \in \mathbb{R}^{2 r_{n}}$, is in the null space of $I-A_{n} D_{n}^{T}$ if and only if $A_{n-1,1} Y_{2}=A_{n-1,2} Y_{1}$.

Proof. For $d=2$ we have $2 r_{n}-r_{n+1}=r_{n-1}$. Therefore, the matrix $Q_{n}$ in the proof of Lemma 2.8 is of size $r_{n-1} \times r_{n-1}$ and invertible. Thus, $V_{n, 2} Y=0$ if and only if $\Xi_{A_{n-1}^{T}}^{T} Y=0$. In this case, $\Xi_{A_{n-1}^{T}}=\left(A_{n-1,2}^{T},-A_{n-1,1}^{T}\right)^{T}$.

Lemma 2.10. Let $A_{n-1, i}$ and $A_{n, i}$ satisfy (2.11). Let $G_{n}$ and $G_{n+1}$ be invertible. If the $Y_{j} \in \mathbb{R}^{r_{n}}, 1 \leq j \leq d$, satisfy

$$
A_{n-1, i} Y_{j}=A_{n-1, j} Y_{i}, \quad 1 \leq i<j \leq d,
$$

then there exists a $Y \in \mathbb{R}^{r_{n+1}}$ such that $Y_{j}=A_{n, j} Y$.

Proof. First we prove that if the $X_{j} \in \mathbb{R}^{r_{n}}$ satisfy $L_{n, i} X_{j}=L_{n, j} X_{i}$, then there is an $X \in \mathbb{R}^{r_{n+1}}$ such that $X_{j}=L_{n+1, j} X$. We can actually define $X$ by

$$
L_{n+1, i} X=X_{i}, \text { or }\left.X\right|_{\mathcal{N}_{n+1, i}}=X_{i} .
$$

Indeed, using $\left.X\right|_{\mathscr{N}_{n+1, i}}=X_{i}$, we have

$$
\left.X\right|_{\mathscr{N}_{n+1, i} \cap \mathscr{N}_{n+1, j}}=L_{n, i} L_{n+1, j} X=L_{n, i} X_{j},
$$

and using $\left.X\right|_{\mathcal{N}_{n+1, j}}=X_{j}$, we have

$$
\left.X\right|_{\mathscr{N}_{n+1, i} \cap \mathscr{N}_{n+1, j}}=L_{n, j} L_{n+1, i} X=L_{n, j} X_{i} .
$$

Therefore, $X$ is well defined. Now let $Y_{j}$ satisfy the assumption. Then by (2.11) we have

$$
L_{n, i} G_{n}^{-1} Y_{j}=L_{n, j} G_{n}^{-1} Y_{i} .
$$

Thus, there is a $Y \in \mathbb{R}^{r_{n+1}}$ such that

$$
L_{n+1, j} G_{n+1} Y=G_{n}^{-1} Y_{j} \text {. }
$$

By (2.11), this is the same as $A_{n, j} Y=Y_{j}$.

2.3. Proof of Theorem 2.1. Suppose that $\left\{\mathbb{P}_{n}\right\}_{n=0}^{\infty}$ is defined recursively by (2.2) and that the matrices $A_{n, i}$ and $B_{n, i}$ satisfy the rank condition (2.4) and the commuting conditions (2.5), (2.6), and (2.7). We shall use induction. For $n=0$ we have $\mathbb{P}_{0}=1, \mathbb{P}_{-1}=0$; thus

$$
\mathbb{P}_{1}=\sum_{i=1}^{d} D_{0, i}^{T} x_{i}-\sum_{i=1}^{d} D_{0, i}^{T} B_{0, i} .
$$

Since $A_{0, i}$ and $D_{0, i}$ are both of size $1 \times d$, we have that $A_{n}$ and $D_{n}$ are both $d \times d$ matrices. It then follows from $D_{0}^{T} A_{0}=I$ that $A_{0} D_{0}^{T}=I$, which implies $A_{0, i} D_{0, j}^{T}=\delta_{i, j}$. Therefore, we have

$$
A_{0, j} \mathbb{P}_{1}=x_{j}-B_{0, j}=x_{j} \mathbb{P}_{0}-B_{0, j} \mathbb{P}_{0} .
$$

We now assume that we have proved that

$$
A_{k, j} \mathbb{P}_{k+1}=x_{j} \mathbb{P}_{k}-B_{k, j} \mathbb{P}_{k}-A_{k-1, j}^{T} \mathbb{P}_{k-1}, \quad 0 \leq k \leq n-1 .
$$


We show that this equation also holds for $k=n$.

From (2.10), (2.2), and (2.3), we get

$$
\begin{gathered}
G_{n+1}=\sum_{i=1}^{d} D_{n, i}^{T} G_{n} L_{n+1, i}, \\
G_{n+1, n}=\sum_{i=1}^{d} D_{n, i}^{T}\left(G_{n, n-1} L_{n, i}-B_{n, i} G_{n}\right), \\
G_{n+1, n-1}=\sum_{i=1}^{d} D_{n, i}^{T}\left(G_{n, n-2} L_{n-1, i}-B_{n, i} G_{n, n-1}-A_{n-1, i}^{T} G_{n-1}\right) .
\end{gathered}
$$

First we prove that for each $j$ the highest three coefficients of

$$
A_{n, j} \mathbb{P}_{n+1}=A_{n, j}\left(G_{n+1} \mathbf{x}^{n+1}+G_{n+1, n} \mathbf{x}^{n}+G_{n+1, n-1} \mathbf{x}^{n-1}+\cdots\right)
$$

are equal to the corresponding coefficients of

$$
x_{j} \mathbb{P}_{n}-B_{n, j} \mathbb{P}_{n}-A_{n-1, j}^{T} \mathbb{P}_{n-1}=Q_{n, j} \mathbf{x}^{n+1}+R_{n, j} \mathbf{x}^{n}+S_{n, j} \mathbf{x}^{n-1}+\cdots,
$$

where

$$
\begin{aligned}
Q_{n, j} & =G_{n} L_{n+1, j}, \\
R_{n, j} & =G_{n, n-1} L_{n, j}-B_{n, j} G_{n}, \\
S_{n, j} & =G_{n, n-2} L_{n-1, j}-B_{n, j} G_{n, n-1}-A_{n-1, j}^{T} G_{n-1} .
\end{aligned}
$$

We need to prove that, for $1 \leq j \leq d$,

$$
\begin{gathered}
A_{n, j} \sum_{i=1}^{d} D_{n, i}^{T} Q_{n, i}=Q_{n, j}, \quad A_{n, j} \sum_{i=1}^{d} D_{n, i}^{T} R_{n, i}=R_{n, j}, \\
A_{n, j} \sum_{i=1}^{d} D_{n, i}^{T} S_{n, j}=S_{n, i} .
\end{gathered}
$$

We can write these equations in a more compact form as

$$
A_{n} D_{n}^{T} Q_{n}=Q_{n}, \quad A_{n} D_{n}^{T} R_{n}=R_{n}, \quad A_{n} D_{n}^{T} S_{n}=S_{n} .
$$

Therefore, we need only prove that the columns of $Q_{n}, R_{n}$, and $S_{n}$ belong to the null space of $I-A_{n} D_{n}^{T}$. But by Lemma 2.8, this reduces to showing that

$$
\begin{gathered}
A_{n-1, i} Q_{n, j}=A_{n-1, j} Q_{n, i}, \quad A_{n-1, i} R_{n, j}=A_{n-1, j} R_{n, i}, \\
A_{n-1, i} S_{n, j}=A_{n-1, j} S_{n, i} .
\end{gathered}
$$

From (2.21), we have that

(2.27) $G_{n-1, n-3} L_{n-2, i}=A_{n-1, i} G_{n, n-2}-B_{n-1, i} G_{n-1, n-2}-A_{n-2, i}^{T} G_{n-2}$.

By (2.22), (2.25), and Lemma 2.3 we get

$$
A_{n-1, i} Q_{n, j}=G_{n-1} L_{n, i} L_{n+1, j}=A_{n-1, j} Q_{n, i} \text {. }
$$


By (2.23), (2.25), (2.26), Lemma 2.3 and (2.6), we have

$$
\begin{aligned}
A_{n-1, i} R_{n, j} & =A_{n-1, i}\left(G_{n, n-1} L_{n, j}-B_{n, j} G_{n}\right) \\
& =G_{n-1, n-2} L_{n-1, i} L_{n, j}-B_{n-1, i} G_{n-1} L_{n, j}-A_{n-1, i} B_{n, j} G_{n} \\
& =G_{n-1, n-2} L_{n-1, i} L_{n, j}-\left(B_{n-1, i} A_{n-1, j}+A_{n-1, i} B_{n, j}\right) G_{n} \\
& =A_{n-1, j} R_{n, i} .
\end{aligned}
$$

Similarly, from (2.24), (2.27), (2.25), (2.26), Lemma 2.3, (2.6), and (2.7) we get

$$
\begin{aligned}
A_{n-1, i} S_{n, j}= & A_{n-1, i}\left(G_{n, n-2} L_{n-1, j}-B_{n, j} G_{n, n-1}-A_{n-1, j}^{T} G_{n-1}\right) \\
= & G_{n-1, n-2} L_{n-2, i} L_{n-1, j}-\left(B_{n-1, i} G_{n-1, n-2}+A_{n-2, i}^{T} G_{n-2}\right) L_{n-1, j} \\
& -A_{n-1, i}\left(B_{n, j} G_{n, n-1}+A_{n-1, j}^{T} G_{n-1}\right) \\
= & G_{n-1, n-2} L_{n-2, i} L_{n-1, j}-B_{n-1, i}\left(A_{n-1, j} G_{n, n-1}+B_{n-1, j} G_{n-1}\right) \\
& -A_{n-2}^{T} A_{n-2, j} G_{n-1}-A_{n-1, i}\left(B_{n, j} G_{n, n-1}+A_{n-1, j}^{T} G_{n-1}\right) \\
= & G_{n-1, n-2} L_{n-2, i} L_{n-1, j}-\left(B_{n-1, i} A_{n-1, j}+A_{n-1, i} B_{n, j}\right) G_{n, n-1} \\
& -\left(A_{n-2}^{T} A_{n-2, j}+B_{n-1, i} B_{n-1, j}+A_{n-1, i} A_{n-1, j}^{T}\right) G_{n-1} \\
= & A_{n-1, j} S_{n, i} .
\end{aligned}
$$

Therefore, we have proved that the highest three coefficient matrices of $A_{n, j} \mathbb{P}_{n}$ and $x_{j} \mathbb{P}_{n}-B_{n, j} \mathbb{P}_{n}-A_{n-1, j}^{T}$ are equal. In other words, we have proved

$$
A_{n, j} \mathbb{P}_{n+1}=x_{j} \mathbb{P}_{n}-B_{n, j} \mathbb{P}_{n}-A_{n-1, j}^{T} \mathbb{P}_{n-1}+\mathbb{Q}_{n-2, j},
$$

where $\mathbb{Q}_{n-2, j} \in \mathbb{R}^{r_{n}}$ are polynomial vectors whose components are elements of $\Pi_{n-2}$.

To complete the proof, we now prove that $\mathbb{Q}_{n-2, i}=0$. Multiplying equation (2.28) by $D_{n, i}^{T}$ and summing up for $1 \leq i \leq d$, from the recurrence formulae (2.2), (2.3), and equation (2.1), we get

$$
\sum_{j=1}^{d} D_{n, j}^{T} \mathbb{Q}_{n-2, j}=\sum_{j=1}^{d} D_{n, j}^{T} A_{n, j} \mathbb{P}_{n+1}-\mathbb{P}_{n+1}=0
$$

On the other hand, from (2.21) and (2.28) we have

$$
\begin{aligned}
A_{n-1, i} \mathbb{Q}_{n-2, j}= & A_{n-1, i}\left(A_{n, j} \mathbb{P}_{n+1}+B_{n, j} \mathbb{P}_{n}+A_{n-1, j}^{T} \mathbb{P}_{n-1}-x_{i} \mathbb{P}_{n}\right) \\
= & A_{n-1, i}\left(A_{n, j} \mathbb{P}_{n+1}+B_{n, j} \mathbb{P}_{n}+A_{n-1, j}^{T} \mathbb{P}_{n-1}\right) \\
& -x_{j}\left(x_{i} \mathbb{P}_{n-1}-B_{n-1, i} \mathbb{P}_{n-1}-A_{n-2, i}^{T} \mathbb{P}_{n-2}\right) \\
= & A_{n-1, i}\left(A_{n, j} \mathbb{P}_{n+1}+B_{n, j} \mathbb{P}_{n}+A_{n-1, j}^{T} \mathbb{P}_{n-1}\right) \\
& -x_{j} x_{i} \mathbb{P}_{n-1}+B_{n-1, i}\left(A_{n-1, j} \mathbb{P}_{n}+B_{n-1, j} \mathbb{P}_{n-1}+A_{n-2, j}^{T} \mathbb{P}_{n-2}\right) \\
& +A_{n-2, i}^{T}\left(A_{n-2, j} \mathbb{P}_{n-1}+B_{n-2, j} \mathbb{P}_{n-2}+A_{n-3, j}^{T} \mathbb{P}_{n-3}\right) \\
= & -x_{i} x_{j} \mathbb{P}_{n-1}+A_{n-1, i} A_{n, j} \mathbb{P}_{n+1} \\
& +\left(A_{n-1, i} B_{n, j}+B_{n-1, i} A_{n-1, j}\right) \mathbb{P}_{n} \\
& +\left(A_{n-1, i} A_{n-1, j}^{T}+B_{n-1, i} B_{n-1, j}+A_{n-2, i}^{T} A_{n-2, j}\right) \mathbb{P}_{n-1} \\
& +\left(A_{n-2, i}^{T} B_{n-2, j}+B_{n-1, i} A_{n-2, j}^{T}\right) \mathbb{P}_{n-2}+A_{n-2, i}^{T} A_{n-3, j}^{T} \mathbb{P}_{n-3} .
\end{aligned}
$$


By the commuting conditions $(2.5),(2.6),(2.7)$, and $x_{i} x_{j} \mathbb{P}_{n-1}=x_{j} x_{i} \mathbb{P}_{n-1}$, it follows that

$$
A_{n-1, i} \mathbb{Q}_{n-2, j}=A_{n-1, j} \mathbb{Q}_{n-2, i}
$$

From (2.28) we get

$$
A_{n, i} G_{n+1}=G_{n} L_{n+1, i}
$$

By Lemma 2.4, $G_{n+1}$ is invertible. Therefore, we can apply Lemma 2.10 to conclude that there is a vector $\mathbb{Q}_{n}$ such that $\mathbb{Q}_{n-2, i}=A_{n, i} \mathbb{Q}_{n}$. It then follows from (2.28) and (2.1) that $\mathbb{Q}_{n}=0$, and thus, $\mathbb{Q}_{n-2, i}=0$. The proof is complete.

\section{GAUSSIAN CUbATURE FormulaE OF DEGREE $2 n-1$}

In this section we consider the application of Theorem 2.1 to the construction of Gaussian cubature formulae of degree $2 n-1$. Let $\mathscr{L}$ be a square positive linear functional. A linear functional

$$
I_{N}(f)=\sum_{k=1}^{N} \lambda_{k} f\left(\mathbf{x}_{k}\right), \quad \lambda_{k}>0, \mathbf{x}_{k} \in \mathbb{R}^{d},
$$

is called a cubature formula of degree $m$, if $\mathscr{L}(f)=I_{N}(f)$ whenever $f \in \Pi_{m}^{d}$, and $\mathscr{L}\left(f^{*}\right) \neq I_{N}\left(f^{*}\right)$ for at least one $f^{*} \in \Pi_{m+1}^{d}$. For fixed $m$ the cubature with a minimal number of nodes $N$ is called the minimal cubature, or the Gaussian cubature. A lower bound for $N$ is [19]

$$
N \geq \operatorname{dim} \Pi_{[m / 2]}^{d} \text {. }
$$

We are interested in Gaussian cubature of degree $2 n-1$, that is, $m=2 n-1$. In this case, the following important result is due to Mysovskikh [13]. In order that there exists a cubature formula which is exact for polynomials in $\Pi_{2 n-1}$ and uses $N=\operatorname{dim} \Pi_{n-1}$ knots, it is necessary and sufficient that $\mathbb{P}_{n}$ has $N$ distinct real zeros. Here, $\mathbb{P}_{n}$ is the vector of multivariate orthogonal polynomials with respect to $\mathscr{L}$ defined by (1.3). A zero of $\mathbb{P}_{n}$ means a common zero of the components of $\mathbb{P}_{n}$.

It is known that all zeros of $\mathbb{P}_{n}$ are real, simple, and distinct. Moreover, $\mathbb{P}_{n}$ has at most $\operatorname{dim} \Pi_{n-1}$ many zeros. Furthermore, the following theorem is true (cf. $[13,18,24])$.

Theorem 3.1. The polynomial vector $\mathbb{P}_{n}$ has $N=\operatorname{dim} \Pi_{n-1}$ distinct zeros if and only if

$$
A_{n-1, i} A_{n-1, j}^{T}=A_{n-1, j} A_{n-1, i}^{T}
$$

for all $1 \leq i, j \leq d$, where the $A_{n-1, i}$ 's are the coefficient matrices in the three-term relation (1.4).

In view of this theorem, $\mathbb{P}_{n}$ does not have $N=\operatorname{dim} \Pi_{n-1}$ zeros in general. Actually, if $\mathscr{L}$ is centrally symmetric, i.e., $\mathscr{L}\left(x^{i} y^{k-i}\right)=0,0 \leq i \leq k$, for all odd $k \in \mathbb{N}$, then Möller [12] proved that for $d=2$ the Gaussian cubature of degree $2 n-1$ does not exist; thus, the corresponding $\mathbb{P}_{n}$ does not have $\operatorname{dim} \Pi_{n-1}$ zeros. On the other hand, a positive example is constructed in [14] for polynomials of degree 5 in two variables. The existence of common zeros 
of multivariate polynomials is in general very difficult to establish. There does not seem to be a general theorem that guarantees a sequence of multivariate polynomials to have a given number of common zeros. However, for orthogonal polynomials we have

Theorem 3.2. Let $A_{n, i}: r_{n} \times r_{n+1}, B_{n, i}: r_{n} \times r_{n}$ be given matrices. Let $\left\{\mathbb{P}_{n}\right\}_{n=0}^{\infty}$ be defined by (2.2) and $\mathbb{P}_{-1}=0, \mathbb{P}_{0}=1$. Then the orthogonal polynomial $\mathbb{P}_{n}$ has exactly $N=\operatorname{dim} \Pi_{n-1}$ distinct real zeros if and only if $B_{n, i}$ is symmetric, $A_{n, i}$ satisfy the rank condition (2.4) and the condition (3.1), and together they satisfy the commuting conditions (2.5), (2.6), and (2.7).

Clearly, this theorem is a consequence of Theorems 2.1 and 3.1. By the Favard theorem, it actually characterizes all possible Gaussian cubatures of degree $2 n-1$ with respect to square positive linear functionals. However, Favard's theorem only establishes the existence of a linear functional $\mathscr{L}$; for practical purposes we would like to know whether there is a measure $\mu$ such that $\mathscr{L} f=\int f d \mu$. From the results in [7], $\mathscr{L}$ always has an integral representation with signed measure. But it may fail to possess an integral representation with nonnegative measure $[3,8]$. For this, we quote the following result from [22], which is proved by using the spectral theorem for a commuting family of selfadjoint operators.

Theorem 3.3. Let $\left\{\mathbb{P}_{n}\right\}_{n=0}^{\infty}, \mathbb{P}_{0}=1$, be a sequence in $\Pi^{d}$. Then the following statements are equivalent:

(i) There exists a nonnegative Borel measure $\mu$ with compact support in $\mathbb{R}^{d}$ such that $\left\{\mathbb{P}_{n}\right\}_{n=0}^{\infty}$ is orthonormal with respect to $\mu$.

(ii) $\left\{\mathbb{P}_{n}\right\}_{n=0}^{\infty}$ satisfies the three-term relation (1.4), the rank condition (1.5), and

$$
\sup _{k \geq 0}\left\|A_{k, i}\right\|<\infty, \quad \sup _{k \geq 0}\left\|B_{k, i}\right\|<\infty,
$$

where $\|\cdot\|$ means the spectral norm for matrices.

The boundedness condition in (ii) can be relaxed to some extent by allowing a noncompactly supported measure in (i) [25]. For practical purposes this theorem is still too abstract. The problem of getting from the coefficients matrices to the measure is certainly a very challenging problem. The importance of our Theorem 3.2 is that it gives a constructive way to generate a sequence of polynomials that has an exact number of real common zeros. That is, if we can find sequences of matrices $\left\{A_{n, i}\right\}$ and $\left\{B_{n, i}\right\}$ that satisfy $(2.4)-(2.7)$ and (3.1), then we can use (2.2) and (2.3) to generate a sequence of polynomials $\left\{\mathbb{P}_{n}\right\} ; \mathbb{P}_{n}$ will have exactly $\operatorname{dim} \Pi_{n-1}$ real, distinct, common zeros. However, the conditions on $A_{n, i}$ and $B_{n, i}$ are quite restrictive. Our next result narrows the possible choices of $A_{n, i}$ and $B_{n, i}$ considerably.

Theorem 3.4. Let $A_{n, i}$ and $B_{n, i}$ satisfy $(2.4),(2.5),(2.6)$, and (2.7). If

$$
B_{n, i} B_{n, j}=B_{n, j} B_{n, i}, \quad 1 \leq i<j \leq d,
$$

then (3.1) cannot be satisfied. For $d=2$ we actually have

$$
\sigma_{n}:=\operatorname{rank}\left(A_{n, 1} A_{n, 2}^{T}-A_{n, 2} A_{n, 1}^{T}\right)=2[(n+1) / 2] .
$$

Proof. Suppose $A_{n, i}$ and $B_{n, i}$ satisfy the given conditions and (3.2). From (2.7) and (3.2) we have

$$
A_{n, i} A_{n, j}^{T}-A_{n, j} A_{n, i}^{T}=-\left(A_{n-1, i}^{T} A_{n-1, j}-A_{n-1, j}^{T} A_{n-1, i}\right) .
$$


Thus, if (3.1) is satisfied, then

$$
A_{n-1, i}^{T} A_{n-1, j}-A_{n-1, j}^{T} A_{n-1, i}=0, \quad 1 \leq i<j \leq d .
$$

From the definition of $\Xi$ in (2.14) and (2.15) we can rewrite these equations as

$$
A_{n-1}^{T} \Xi_{A_{n-1}}=0 \text {. }
$$

Thus, the columns of $\Xi_{A_{n-1}}$ belong to the null space of $A_{n-1}^{T}$. By Theorem 2.1 we can construct $\mathbb{P}_{n}$ using $A_{n, i}$ and $B_{n, i}$, and by Lemma 2.4, the leading coefficient matrix $G_{n}$ of $\mathbb{P}_{n}$ is invertible. Therefore, as in the proof of Lemma 2.8, we have from (2.12) and Lemma 2.7 that

$$
\operatorname{rank} \Xi_{A_{n-1}}=\operatorname{rank} \Xi_{L_{n}}=d r_{n-1}-r_{n-2} \text {. }
$$

On the other hand, we have from (2.5) that $A_{n-1}^{T} \Xi_{A_{n-2}^{T}}=0$, and it follows from (2.4) and (2.20) that the columns of $\Xi_{A_{n-2}^{T}}$ form a basis for the null space of $A_{n-1}^{T}$. Therefore,

$$
d r_{n-1}-r_{n-2}=\operatorname{rank} \Xi_{A_{n-1}} \leq \operatorname{rank} \Xi_{A_{n-2}^{T}}=d r_{n-1}-r_{n} .
$$

This is possible only for $d=1$ since $r_{n-2}<r_{n}$ for $d \geq 2$.

We now prove (3.3). Let $\Lambda_{n}:=A_{n, 1} A_{n, 2}^{T}-A_{n, 2} A_{n, 1}^{T}$. Since $\Lambda_{n}$ is skewsymmetric, we know that rank $\Lambda_{n}$ is an even integer. We consider the null space of $\Lambda_{n}$. If $\mathbf{x}_{n+1} \in \mathbb{R}^{r_{n+1}}$ satisfies

$$
\left(A_{n+1,1} A_{n+1,2}^{T}-A_{n+1,2} A_{n+1,1}^{T}\right) \mathbf{x}_{n+1}=0,
$$

then, by (3.4),

$$
\left(A_{n, 1}^{T} A_{n, 2}-A_{n, 2}^{T} A_{n, 1}\right) \mathbf{x}_{n+1}=0
$$

Hence,

$$
\Xi_{A_{n}}=\left[\begin{array}{cc}
O & I \\
-I & O
\end{array}\right]\left(\begin{array}{l}
A_{n, 1} \\
A_{n, 2}
\end{array}\right)=: T_{n} A_{n}
$$

and

$$
A_{n}^{T} T_{n} A_{n} \mathbf{x}_{n+1}=0 .
$$

This shows that $T_{n} A_{n} \mathbf{x}_{n+1}$ is in the null space of $A_{n}^{T}$. Since the columns of $T_{n}\left(A^{T}\right)_{n-1}$ span the null space of $A_{n}^{T}$, there exists $\mathbf{x}_{n-1} \in \mathbb{R}^{r_{n-1}}$ such that $T_{n} A_{n} \mathbf{x}_{n+1}=T_{n}\left(A^{T}\right)_{n-1} \mathbf{x}_{n-1}$. Since $T_{n}$ is clearly invertible, we actually have $A_{n} \mathbf{x}_{n+1}=\left(A^{T}\right)_{n-1} \mathbf{x}_{n-1}$ or

$$
\mathbf{x}_{n+1}=D_{n}^{T}\left(A^{T}\right)_{n-1} \mathbf{x}_{n-1}
$$

by (2.1). From (2.5) it follows that $\left(A_{n-1,1}, A_{n-1,2}\right) T_{n} A_{n}=0$. Thus,

$$
\left(A_{n-1,1} A_{n-1,2}^{T}-A_{n-1,2} A_{n-1,1}^{T}\right) \mathbf{x}_{n-1}=0 .
$$

Equations (3.5), (3.6), and (3.7) enable us to use induction. Indeed, since $\Lambda_{0}$ is of size $1 \times 1$ and skew-symmetric, we have $\Lambda_{0}=0$. Thus, rank $\Lambda_{0}=0$. For $n=1$ we have from (3.4) that $\Lambda_{1}=A_{0}^{T} T_{0} A_{0}$, from which it follows that $\Lambda_{1}$ is invertible. Therefore, rank $\Lambda_{1}=2$. Hence, rank $\Lambda_{2 m}=2 m$ and $\operatorname{rank} \Lambda_{2 m+1}=2 m+2$.

For $d=2$ the rank equation (3.3) was first proved by Möller for the case $B_{n, i}=0$. In [24] it is shown that $\mathbb{P}_{n}$ can have at most $N-\sigma_{n}$ zeros. Our proof 
of (3.3) follows the approach used in [18]. For a given linear functional $\mathscr{L}$ on $\Pi^{d}$, Theorem 3.4 shows that if $\mathscr{L}\left(x_{1} \mathbb{P}_{k} \mathbb{P}_{k}^{T}\right)=\mathscr{L}\left(x_{2} \mathbb{P}_{k} \mathbb{P}_{k}^{T}\right)$, then Gaussian cubature of degree $2 n-1$ is not possible. Actually, for most classical functionals, Gaussian cubature of degree $2 n-1$ does not exist. Examples of these functionals include all those considered in [9], in particular, the integrals with respect to the Jacobi weights on a rectangle, simplex, or sphere $([1,2])$. On the other hand, there are square positive linear functionals that lead to Gaussian cubature of degree $2 n-1$; see $[14,10]$. Our Theorem 3.2 provides a method to generate these cubatures, or rather, the nodes that these cubatures are based on. The difficult question remains of how to find the measure $\mu$ from the coefficient matrices of the three-term relation. In view of the importance of Gaussian cubatures in analysis, it can be expected that those measures for which Gaussian cubatures exist may have some interesting and peculiar properties.

\section{ACKNOWLEDGMENT}

The author thanks the referee for his valuable comments that improved the presentation of this paper.

\section{BIBLIOGRAPHY}

1. H. Berens and H. Schmid, On the number of nodes of odd degree cubature formulae for integrals with Jacobi weights on a simplex, Numerical Integration (T. O. Espelid and A. Genz, eds.), Kluwer Academic Publishers, Dordrecht, 1992, pp. 37-44.

2. $\mathrm{H}$. Berens, $\mathrm{H}$. Schmid, and $\mathrm{Y}$. Xu, A note on two-dimensional definite orthogonal systems and on a lower bound for the number of nodes of associated cubature formulae, SIAM J. Math. Anal. (to appear).

3. C. Berg, J. P. R. Christensen, and C. U. Jensen, A remark on the multidimensional moment problem, Math. Ann. 243 (1979), 163-169.

4. T. S. Chihara, An introduction to orthogonal polynomials, Math. Appl., vol. 13, Gordon and Breach, New York, 1978.

5. R. A. Horn and C. R. Johnson, Matrix analysis, Cambridge Univ. Press, New York, 1985.

6. M. A. Kowalski, The recursion formulas for orthogonal polynomials in $n$ variables, SIAM J. Math. Anal. 13 (1982), 309-315.

7. Orthogonality and recursion formulas for orthogonal polynomials in $n$ variables, SIAM J. Math. Anal. 13 (1982), 316-323.

8. Algebraic characterization of orthogonality in the space of polynomials, Lecture Notes in Math., vol. 1171, Springer-Verlag, New York and Berlin, 1985, pp. 101-110.

9. H. L. Krall and I. M. Sheffer, Orthogonal polynomials in two variables, Ann. Mat. Pura Appl. (4) 76 (1967), 325-376.

10. V. A. Kuzmenkov, The existence of cubature formulas with the least possible number of nodes, Zh. Vychisl. Mat. i Mat. Fiz. 16 (1976), 1337-1339.

11. H. Möller, Polynomideale und Kubaturformeln, Thesis, Univ. Dortmund, 1973.

12. __ Kubaturformeln mit minimaler Knotenzahl, Numer. Math. 25 (1976), 185-200.

13. I. P. Mysovskikh, Numerical characteristics of orthogonal polynomials in two variables, Vestnik Leningrad Univ. Math. 3 (1976), 323-332.

14. I. P. Mysovskikh and V. Ya. Chernitsina, Answer to a question of Radon, Dokl. Akad. Nauk SSSR 198 (1971), 537-539.

15. J. Riordan, Combinatorial identities, Wiley, New York, 1968.

16. H. Schmid, On cubature formulae with a minimal number of knots, Numer. Math. 31 (1978), 282-297.

17. __ Interpolatorische Kubaturformeln, Dissertationes Math. 210 (1983), 1-122. 
18. __ Minimal cubature formulae and matrix equation, preprint.

19. A. Stroud, Approximate calculation of multiple integrals, Prentice-Hall, Englewood Cliffs, NJ, 1971.

20. G. Szegö, Orthogonal polynomials, 4th ed., Amer. Math. Soc. Colloq. Publ., vol. 23, Amer. Math. Soc., Providence, RI, 1975.

21. Y. Xu, On multivariate orthogonal polynomials, SIAM J. Math. Anal. 24 (1993), 783-794.

22. _ Multivariate orthogonal polynomials and operator theory, Trans. Amer. Math. Soc. (to appear).

23. $\_$, Gaussian cubature and bivariate polynomial interpolation, Math. Comp. 59 (1992), 547-555.

24. _ Block Jacobi matrices and zeros of multivariate orthogonal polynomials, Trans. Amer. Math. Soc. (to appear).

25. __ Unbounded commuting operators and multivariate orthogonal polynomials, Proc. Amer. Math. Soc. (to appear).

Department of Mathematics, University of Oregon, Eugene, Oregon 97403-1222

E-mail address: yuan@bright.uoregon.edu 